\title{
Effect of grain size distribution on california bearing ratio (CBR) and modified proctor parameters for granular materials
}

\author{
Autores \\ Jose Duque, William Fuentes, Silvia Rey, Enois Molina
}

\begin{abstract}
The California bearing ratio (CBR) and modified proctor parameters (maximum dry unit weight $y d(\max ) y d(\max )$ and optimum moisture content woptwopt) are valuable indicators of the compaction quality of subgrades, embankments and granular fills. In the engineering practice, correlations of these variables with granulometric properties of the soil are required, especially since testing for these variables can be time-consuming when a large number of samples are analyzed. In this work, 20 different granular materials with varying grain size distributions were prepared and tested. Their grain size distribution properties and their parameters $C B R, y d(\max ) y d(\max )$ and woptwopt were determined. These results were analyzed along with a compilation of 77 additional experimental results on granular materials reported in the literature. The influence of some granulometric properties on the parameters CBR, $y d(\max ) y d(\max )$ and woptwopt was statistically examined, and some correlations were proposed for these variables. Subsequently, it was demonstrated that the proposed correlations show better accuracy tother reported correlations in the literature. Finally, this work ends with some concluding remarks.
\end{abstract}

Palabras clave

California bearing ratio (CBR), Grain size distribution, Modified proctor, Granular soils 